\title{
Vascular co-option in lung cancer metastatic to the eye after treatment with bevacizumab
}

\author{
Efrem D. Mandelcorn • Alan G. Palestine • \\ Sandor Dubovy $\cdot$ Janet L. Davis
}

Received: 23 September 2010 / Accepted: 26 October 2010 /Published online: 17 November 2010

(C) The Author(s) 2010. This article is published with open access at Springerlink.com

\begin{abstract}
Background Chemotherapy with bevacizumab alters the angiogenic environment, and therefore, the growth and spread of metastases. We present a patient with metastatic lung adenocarcinoma to the eye with findings suggestive of retinal vascular co-option.

Methods Case report.

Results A 57-year-old male, receiving systemic bevacizumab for metastatic lung adenocarcinoma, presented with vitreous opacities and clumped deposits adherent to the retinal vessels. No choroidal metastases were present. Diagnostic vitrectomy yielded cellular evidence of adenocarcinoma, with thyroid transcription factor-1 staining confirming a lung primary. Conclusion The perivascular growth of small foci of metastatic vitreous cells suggests vascular co-option from the native retinal circulation. Similar modification of metastatic disease by bevacizumab has been observed in animal models and selected human cases.
\end{abstract}

This paper was presented at the American Uveitis Society Meeting, Fort Lauderdale, Florida, USA, May 1, 2010

This study was approved as exempted by the Human Subjects Research Committee, University of Miami Miller School of Medicine.

E. D. Mandelcorn $\cdot$ S. Dubovy $\cdot$ J. L. Davis

Department of Ophthalmology, University of Miami School of Medicine, Bascom Palmer Eye Institute,

Miami, FL, USA

\section{A. G. Palestine \\ Retina Consultants, \\ Bethesda, MD, USA}

E. D. Mandelcorn $(\square)$

Department of Ophthalmology \& Vision Sciences Toronto Western Hospital, University Health Network, University of Toronto,

399 Bathurst Street, 6E-415,

Toronto M5T 2S8 Ontario, Canada

e-mail: efrem.mandelcorn@utoronto.ca
Keywords Angiogenesis - Bevacizumab - Metastatic lung carcinoma $\cdot$ Vascular co-option $\cdot$ Retina $\cdot$ Vitreous

\section{Introduction}

Ocular metastases most commonly present as mass lesions in the choroid. Retinal metastases and diffuse metastases to the vitreous have been previously reported in cutaneous melanoma, and breast and lung adenocarcinoma without any recognizable angiocentric pattern. We present a patient with retinal and vitreous metastases from lung adenocarcinoma whose ocular findings suggest that bevacizumab treatment resulted in distinctive perivascular growth by fostering vascular co-option of the retinal vasculature by metastatic tumor cells.

\section{Case report}

We examined a 57-year-old male who presented to the Bascom Palmer Eye Institute in Oct 2009 with a 6-month history of floaters in his left eye (OS). He had reported flashes in the OS 3 months prior and had undergone laser retinopexy without resolution of symptoms and was now diagnosed as uveitis. Past medical history was significant for stage IV non-small cell carcinoma of the lung diagnosed in Aug 2005 and treated with left pneumonectomy. Within 1 year, brain metastases developed and were treated with whole brain irradiation, 6-thioguanine, temozolomide, and stereotactic radiosurgical treatment of some larger masses. In June 2009, recurrent brain metastases were detected. Cerebrospinal fluid was negative for malignant cells. Chemotherapy was resumed. Erlotinib was stopped 2 months prior to presentation, and a 6 -week prior MRI 
of the brain showed no evidence of tumor. Treatment with bevacizumab, capecitabine, and camptosar was ongoing. $\mathrm{He}$ had an implantable venous access device in the right subclavian vein present for 4 years. The skin over the device was intact and uninflamed. He was afebrile.

Vision was 20/15 in the right eye (OD) and 20/40 OS. Pupil exam was normal with a $10 \%$ subjective reduction in light brightness OS. There was mild conjunctival injection in the OS more than in the OD. He had 1+ anterior chamber cell OS and no cell OD. There were no posterior synechiae. Examination of the vitreous and retina OD was entirely normal. In the OS, there were $2+$ vitreous cells, with clumping (Fig. 1). The cells appeared to ramify on the surface of the retina with well-defined cellular clumps along the retinal arteries and veins (Fig. 2). Optical coherence tomography confirmed a preretinal location (Fig. 2).

Fungal endophthalmitis was considered. A diagnostic pars plana vitrectomy was performed with intravitreal injection of $5 \mu \mathrm{g}$ amphotericin. The vitreous specimen was sent for histopathology and culture. No organisms were identified on gram stain. Corynebacterum grew from a single medium used to culture the vitreous and was interpreted as a contaminant. Blood and urine cultures were negative. Millipore preparations of the vitreous specimen revealed cellular evidence of adenocarcinoma (Fig. 3). Immunocytochemistry was positive for TTF-1(thyroid transcription factor-1) indicating a lung primary (Fig. 3).

The patient was referred to his oncologist for further evaluation and treatment. Temozolomide was added. $\mathrm{He}$ relocated to Maryland. In March 2010, multiple lymph nodes showed metastatic disease on PET scan, and he was switched to single agent chemotherapy with gemcitabine. Vision was 20/70. Some cellular clumps had resolved, and there were other new ones, including a large peripapillary lesion (Fig. 4), which remained stable in size over the next 2 months. The right eye remained clinically uninvolved.

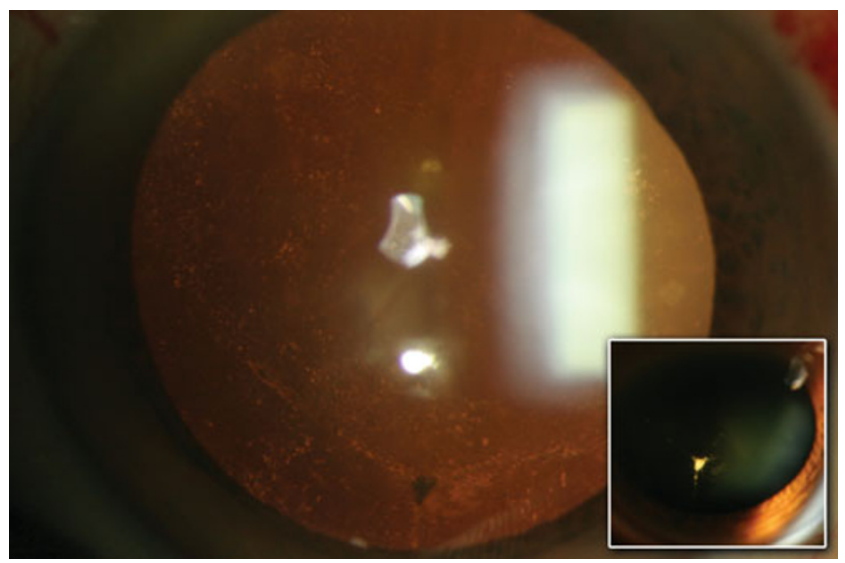

Fig. 1 Slit-lamp biomicroscopy OS demonstrating vitreous cell in retroillumination behind the lens. The inset shows a large cluster of cells in the anterior vitreous

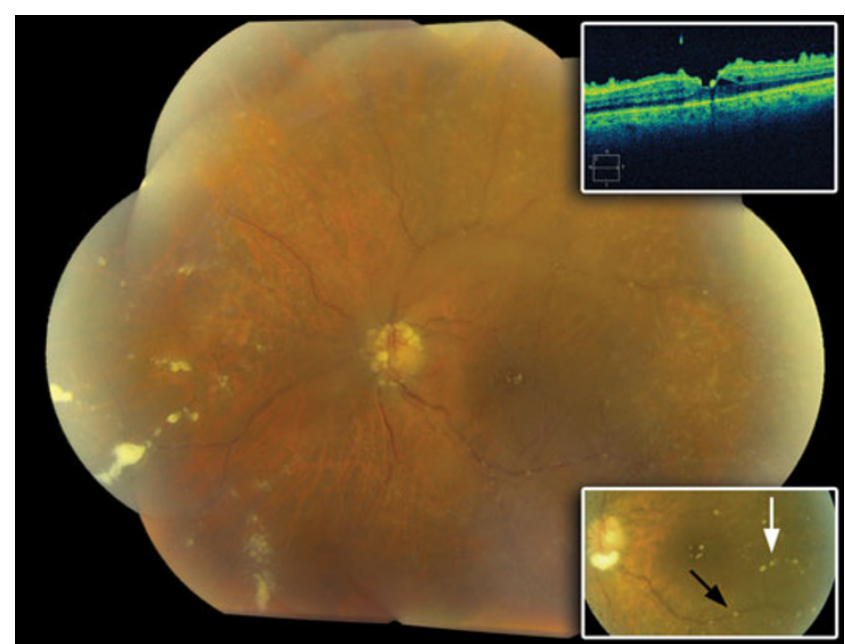

Fig. 2 Fundus photo OS demonstrating a reticular pattern of vitreous cellular clumping associated with the retinal vasculature, both arteriole (white arrow) and venous (black arrow); Upper Inset: OCT OS demonstrating preretinal vitreous cellular clumping

\section{Discussion}

Lung cancer is the most common origin of ocular metastases. Malignant cells can metastasize via the systemic circulation to the high-flow posterior ciliary circulation and seed the choroid. Retinal metastases can shed into the vitreous, or the vitreous can be seeded via the highly vascular ciliary processes of the ciliary body. In cases of central nervous system malignancy, it is hypothesized that cells may gain access to the vitreous via the optic nerve. A Medline search revealed two prior reports of lung cancer metastasizing to the vitreous that were confirmed by cytologic examination and cytokeratin staining. [1,2] Specific TTF-1 immunocy-

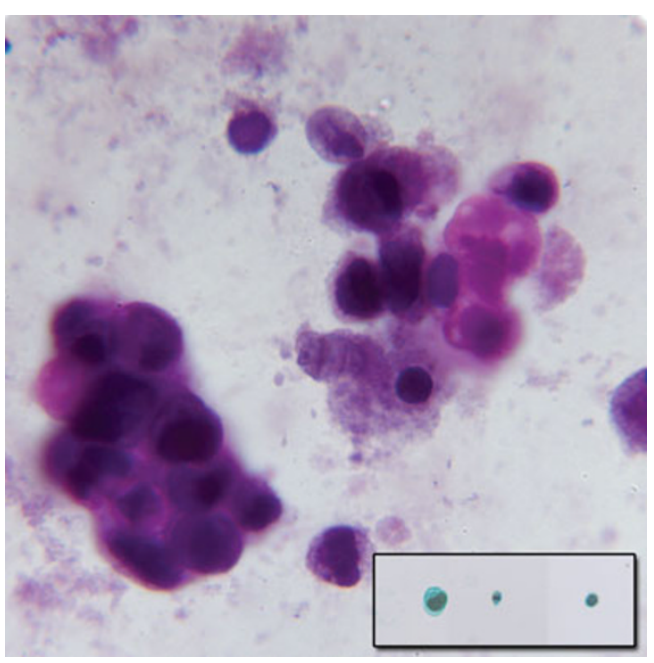

Fig. 3 H\&E stain of vitreous specimen showing atypical epithelial cells with large and prominent nuclei arranged in small clumps $(\times 100)$; Inset: TTF-1 stain of vitreous cells $(\times 40)$ 
Fig. 4 Fundus photo, red-free photo, and fluorescein angiogram 5 months post-vitrectomy OS demonstrating a new large clump of tumor cells at the optic nerve head and new cellular clumps along the retinal arteries and veins. There is both late arterial and venous leakage, as well as macular and optic nerve leakage
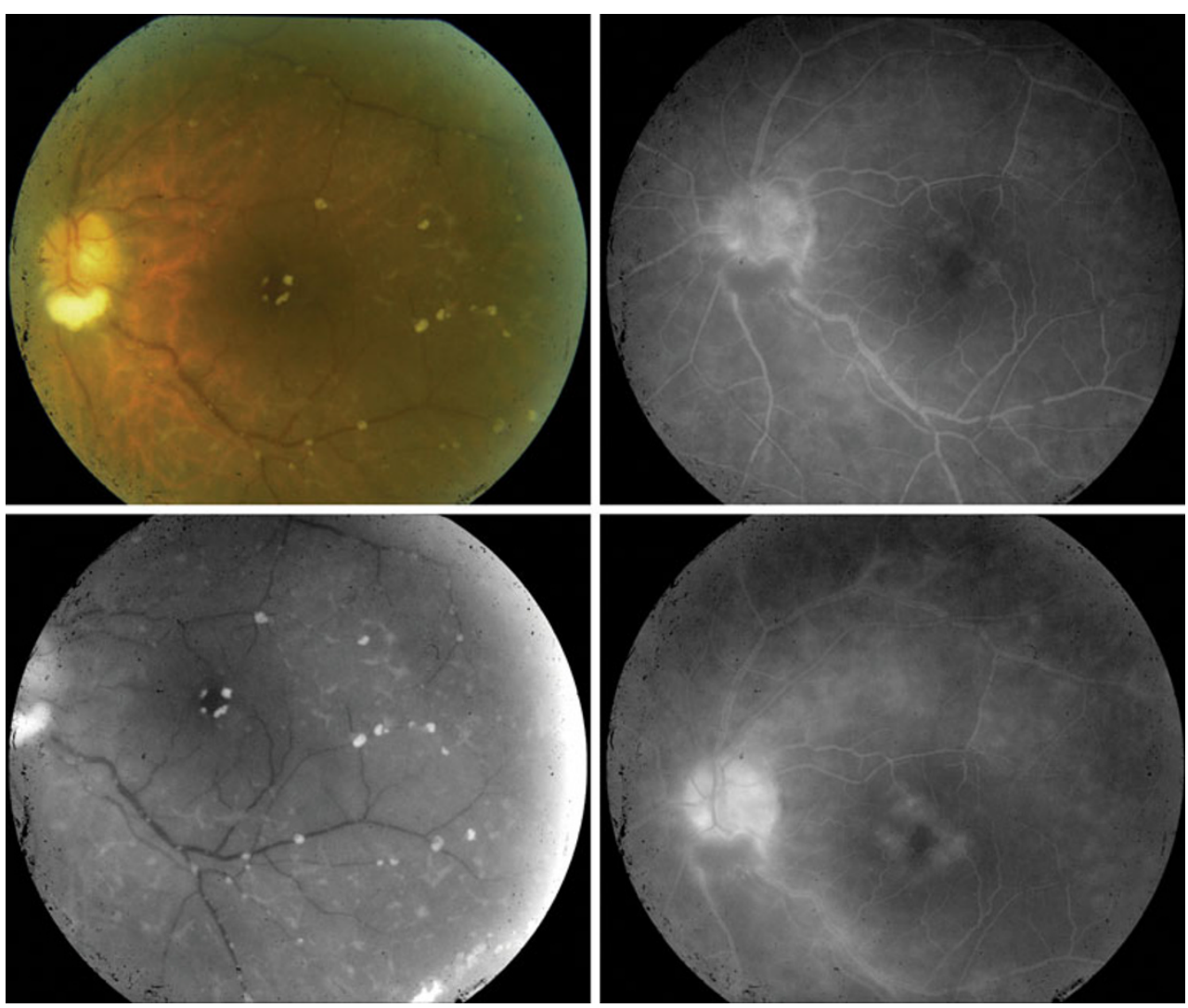

tochemical staining [3] provides an additional tool for diagnosis of vitreous metastasis from a lung primary.

Preoperative OCT examination confirmed preretinal localization of the cellular deposits. The striking finding at surgery was the adherence of individual cellular clumps to the retinal surface often lining up along the retinal arteries and veins. Settling of cells into areas of tight vitreous insertion around the optic nerve or along the large retinal vessels was unlikely, since the deposits did not change after inducing a posterior vitreous detachment. The deposits could be individually aspirated from the retinal surface and did not form a sheet as would occur if the cells were growing along the posterior hyaloid.

We propose that the retinal adherence and the predilection for growth along the retinal vessels represent vascular co-option by tumor cells in an anti-angiogenic milieu created by systemic bevacizumab. A solid tumor will not generally exceed a diameter of $0.4-1.0 \mathrm{~mm}$ without first establishing its own vasculature, [4] which increases proportionate to tumor size. Use of vascular endothelial growth factor (VEGF) inhibitors, such as bevacizumab, thus arrest tumor growth. For comparison, the width of the optic nerve is approximately $1.5 \mathrm{~mm}$ and the width of a large retinal vein about $0.150 \mu \mathrm{m}$. The large optic nerve deposit seen in March 2010 was roughly $1.0 \mathrm{~mm}$ in greatest dimension (Fig. 3), which may have grown to this extent only because of co-option of vascular supply from the oxygen-rich vessels on the nerve face; it may have been unable to increase further in size without angiogenesis.

Survival of tumor cells by vascular co-option in an antiVEGF environment has been demonstrated in animal studies [5]. In a study by Rubenstein et al. [5], G55 human glioblastoma cells were inoculated into the basal ganglia of athymic rats which were then treated with intraperitoneal anti-VEGF antibody. There was a statistically significant increase in survival in the anti-VEGF treated animals compared to untreated controls. Tumors in the untreated animals grew as well-circumscribed, focal lesions, whereas tumors in the anti-VEGF treated animals had an infiltrative and invasive pattern of growth. Ninety per cent of these small $(10 \mu \mathrm{m})$ "satellites" were located along a blood vessel, which was attributed to vascular co-option in an antiangiogenic environment. TUNEL staining indicated higher apoptosis indices in tumor cells remote from blood vessels compared to tumor cells co-opting the native circulation.

In humans, vascular co-option is also likely to exist. Two patients with non-small cell lung carcinoma on systemic bevacizumab developed atypical leptomeningeal carcinomatosis identified by both neuroradiological imaging and histopathology [6]. Both cases demonstrated nodular micrometastases centered on small blood vessels suggesting cooption of the normal vasculature. 


\section{Conclusion}

In summary, we have identified a 57-year-old male on systemic bevacizumab with vitreous metastasis of TTF-1 positive metastatic non-small cell lung adenocarcinoma with tumor cell clumping along the retinal vasculature. We believe that this distribution of cells demonstrates retinal vascular co-option in an anti-angiogenic environment similar to that shown in previous animal and human studies.

Funding No grants or funds were used to support this study.

Conflict of interest The authors have no proprietary interest in this report.

Open Access This article is distributed under the terms of the Creative Commons Attribution Noncommercial License which per- mits any noncommercial use, distribution, and reproduction in any medium, provided the original author(s) and source are credited.

\section{References}

1. Gündüz K, Shields JA, Shields CL, Eagle RC Jr, Ehya H, McLaughlin W Jr (1998) Lung carcinoma metastatic to the vitreous cavity. Retina 18(3):285-286

2. Latkany P, Ciulla TA, Younger C, Gao H (2000) Nonsmall cell lung adenocarcinoma metastatic to the vitreous without fundus lesions. Ophthalmic Surg Lasers 31(2):155-6

3. Pelosi G, Fraggetta F, Pasini F et al (2001) Immunoreactivity for thyroid transcription factor-1 in stage I non-small cell carcinomas of the lung. Am J Surg Pathol 25(3):363-372

4. Hanahan D, Folkman J (1996) Patterns and emerging mechanisms of the angiogenic switch during tumorigenesis. Cell 86(3):353-364

5. Rubenstein JL, Kim J, Ozawa T et al (2000) Anti-VEGF antibody treatment of glioblastoma prolongs survival but results in increased vascular cooption. Neoplasia 2:306-314

6. Kleinschmidt-DeMasters BK, Damek DM (2010) The imaging and neuropathological effects of Bevacizumab (Avastin) in patients with leptomeningeal carcinomatosis. J Neurooncol 96(3):375-384 